\title{
The RISCK food-exchange model: a dietary strategy to alter the amount and composition of fat and carbohydrate in free-living individuals
}

\author{
Carmel Moore ${ }^{1}$, Hannah Farrant ${ }^{1}$, Rachel Gitau ${ }^{2}$, Louise Goff ${ }^{3}$, Margaret Griffin ${ }^{4}$, Fiona Lewis ${ }^{5}$ \\ and Julie Lovegrove ${ }^{2}$ \\ ${ }^{1}$ MRC Human Nutrition Research, Cambridge, UK, ${ }^{2}$ University of Reading, Reading, UK, ${ }^{3}$ Imperial College, London, UK, \\ ${ }^{4}$ University of Surrey, Guildford, UK and ${ }^{5}$ King's College, London, UK
}

The metabolic syndrome (MS) affects as many as $25 \%$ of the UK population ${ }^{(1)}$ and confers substantial risk of CVD. A key scientific and public health question is whether reducing intakes of SFA via low-fat-high-carbohydrate (CHO) diets or by moderate-fat diets in which SFA are substituted with MUFA have differential effects on risk factors for MS. Moreover, a further question to be addressed is the type of dietary $\mathrm{CHO}$, and there is current interest in the role of foods with a low glycaemic index (GI).

RISCK is a multi-centre randomised controlled dietary intervention study of 720 subjects at increased risk of MS. The study design has been reported previously ${ }^{(2)}$. All subjects followed a 4-week run-in diet (high-SFA (HS)-high-GI (HGI)). They continued on this diet for a further 24 weeks or followed one of four isoenergetic interventions: high-MUFA (HM)-HGI; HM-low-GI (LGI); low-fat (LF)-HGI; LFLGI. A food-exchange model was developed to implement each diet, facilitated by the supply of specifically-formulated or selected foods ${ }^{(3)}$. Dietary records (4d) during run-in and at 24 weeks were used to assess compliance with the intervention strategy.

A total of 548 subjects completed the study. Reported intakes showed that the dietary strategy was largely successful in meeting target intakes (see Table). During the intervention differences in dietary fat and CHO (LF $v$ HS and HM) and SFA (HS $v$ HM and LF) and MUFA (HM $v$ HS and LF) were highly significant $(P<0.001)$. An inadvertent small, but significant, increase in $n-6$ PUFA was shown in the HM groups, primarily as a result of prescribed increases in nut intake. The GI difference between the HGI and LGI groups was slightly smaller than predicted, but significantly lower in the LGI group than the HGI group $(P<0.001)$.

Table. Intakes ( $\%$ food energy) during run-in and on each intervention diet compared with RISCK target intakes (T)

\begin{tabular}{|c|c|c|c|c|c|c|c|c|c|c|c|c|c|c|c|c|}
\hline & \multicolumn{5}{|c|}{ Run-in } & \multicolumn{10}{|c|}{ Intervention } & \multirow[b]{3}{*}{$P<\dagger$} \\
\hline & \multicolumn{2}{|c|}{ HS-HGI ( $n$ 511) } & \multicolumn{2}{|c|}{ HS-HGI $(n$ 80) } & \multirow[b]{2}{*}{$\mathrm{T}$} & \multicolumn{2}{|c|}{ HM-HGI $(n$ 103) } & \multicolumn{2}{|c|}{ HM-LGI $(n$ 102) } & \multirow[b]{2}{*}{$\mathrm{T}$} & \multicolumn{2}{|c|}{ LF-HGI ( $n$ 105) } & \multicolumn{2}{|c|}{ LF-LGI ( $n$ 108) } & \multirow[b]{2}{*}{$\mathrm{T}$} & \\
\hline & Mean & SD & Mean & SD & & Mean & $\mathrm{SD}$ & Mean & $\mathrm{SD}$ & & Mean & SD & Mean & $\mathrm{SD}$ & & \\
\hline Fat & 39.1 & 5.5 & 39.3 & 6.2 & 38 & 37.4 & 7.0 & 35.7 & 6.0 & 38 & 28.8 & 7.0 & 27.1 & 6.6 & 28 & $\overline{0.001}$ \\
\hline SFA & 17.0 & 3.1 & 16.9 & 3.1 & 18 & 10.1 & 2.6 & 9.4 & 2.6 & 10 & 9.4 & 2.9 & 8.5 & 2.6 & 10 & 0.001 \\
\hline PUFA & 6.2 & 1.9 & 6.3 & 1.9 & 6 & 7.1 & 1.9 & 7.1 & 2.1 & 6 & 5.8 & 2.0 & 5.5 & 2.0 & 6 & 0.001 \\
\hline MUFA & 12.0 & 2.2 & 12.2 & 2.8 & 12 & 16.9 & 4.9 & 16.3 & 4.0 & 20 & 10.4 & 3.3 & 10.0 & 3.1 & 11 & 0.001 \\
\hline $\mathrm{CHO}$ & 44.3 & 5.8 & 43.0 & 6.3 & 45 & 45.7 & 6.8 & 47.0 & 6.6 & 45 & 52.3 & 7.2 & 53.7 & 7.0 & 55 & 0.001 \\
\hline GI & 63.5 & 3.6 & 64.6 & 3.6 & - & 63.2 & 3.7 & 55.0 & 3.7 & $\mathrm{C}-11^{*}$ & 64.8 & 3.8 & 56.0 & 3.4 & $\mathrm{E}-13$ & 0.001 \\
\hline
\end{tabular}

*Global test (Kruskal Wallis; $4 \mathrm{df}$ ) of differences between treatments during the intervention

$\dagger$ Target difference between HGI and LGI diets.

The RISCK food-exchange model was a broadly effective dietary strategy for the implementation of five experimental diets with specific targets for fat and $\mathrm{CHO}$ intake in a free-living population.

The RISCK study was funded by the Food Standards Agency (NO2031).

1. Tonkin $\mathrm{R}$ (2003) The $\mathrm{X}$ factor: obesity and the metabolic syndrome. http://www.the-ba.net/NR/rdonlyres/9EE73A6C-C61F-4007-93732BCBFC08F62B/0/Report.pdf

2. Jebb SA, Frost G, Griffin BA, Lovegrove JA, Moore C, Sanders T \& Williams CM (2007) Nutr Bull 32, 154-156.

3. Moore C, Gitau R, Frost G, Griffin BA, Jebb SA, Sanders TS \& Lovegrove JA (2007) Proc Nutr Soc 65, 86A. 